\title{
Short-term recognition memory impairment is associated with decreased expression of FK506 binding protein 51 in the aged mouse brain
}

\author{
Virawudh Soontornniyomkij • Victoria B. Risbrough • Jared W. Young • \\ Chelsea K. Wallace • Benchawanna Soontornniyomkij • Dilip V. Jeste • \\ Cristian L. Achim
}

Received: 4 January 2010 /Accepted: 29 March 2010/Published online: 27 April 2010

(C) The Author(s) 2010. This article is published with open access at Springerlink.com

\begin{abstract}
Evidence suggests that increased glucocorticoid receptor (GR) signaling may contribute to cognitive decline with age. We hypothesized that alterations in GR signaling pathway molecules, FK506 binding protein (FKBP) 51 and FKBP52, were associated with memory impairment in aged mice. We used the singletrial object recognition test to measure short-term memory in 18 aged mice compared to 22 young mice, and employed quantitative immunohistochemistry to assess cellular expression of those three proteins in the frontal cortex, hippocampal CA1, and dentate gyrus. Values of the discrimination ratio (DR, a measure of novelty preference) in aged mice were significantly lower than those in young mice (mean 0.54 vs. $0.67, p=$ $0.003, t$ test). Aged mice with DR below 0.54 were considered impaired $(n=9)$. In the three neuroanatomic regions studied, the immunoreactivity normalized to
\end{abstract}

V. Soontornniyomkij · V. B. Risbrough · J. W. Young •

C. K. Wallace $\cdot$ B. Soontornniyomkij • D. V. Jeste $\cdot$

C. L. Achim

Sam and Rose Stein Institute for Research on Aging,

University of California, San Diego,

9500 Gilman Drive, La Jolla,

CA 92093-0603, USA

V. Soontornniyomkij $(\bowtie) \cdot$ V. B. Risbrough •

J. W. Young - C. K. Wallace - B. Soontornniyomkij •

D. V. Jeste - C. L. Achim

Department of Psychiatry, School of Medicine,

University of California, San Diego,

9500 Gilman Drive, La Jolla,

CA 92093-0603, USA

e-mail: vsoontor@ucsd.edu the area measured (IRn) for GR was significantly increased in aged mice regardless of their task performance compared to young mice $(p<0.005)$, as was the FKBP52 IRn $(p<0.007, U$ test). In the frontal cortex and CA1, the FKBP51 IRn was significantly lower in impaired aged mice than in unimpaired aged mice ( $p<0.01$ and $<0.05$, respectively) and in young mice ( $p<0.05$ and $<0.01$, respectively, Dunn's post hoc test). In aged mice, the frontal cortex FKBP51 IRn correlated directly with DR $\left(r_{\mathrm{s}}=0.68, p=0.002\right.$, Spearman rank correlation). These observations suggest that recognition memory impairment in aged mice is associated with decreased FKBP51 expression that may promote GR-mediated glucocorticoid signaling to a greater extent than in unimpaired aged mice.

Keywords Aging · Brain immunophilins · FKBP51 · FKBP52 - Glucocorticoid receptor signaling ·

Object recognition test

\section{Introduction}

Cognitive and affective functioning is considered to be one of the most important determining factors in successful aging (Depp et al. 2007). Although cognitive decline affects a large proportion of the aging population, the severity displays a great individual variation and there are elderly people who do not exhibit cognitive deterioration. The differential susceptibility to the development of aging-related cognitive impairment 
may be driven by genetic polymorphisms, epigenetic phenomena, and dissimilar life-long environmental exposure to stressors (Goosens and Sapolsky 2007). Aging-related cognitive decline is different from neurodegenerative disorders like Alzheimer's disease in terms of more severity and more rapid progression, as well as the presence of substantial neuronal loss, in the latter. Neurobiological substrates contributing to cognitive impairment in old age largely remain unclear.

The hypothalamic-pituitary-adrenal (HPA) axis activity is one of several proposed mechanisms involved in brain aging. While there are considerable individual differences in circadian cortisol levels with age, excessive circulating levels of glucocorticoids associated with exogenous administration, Cushing's syndrome, or HPA axis hyperactivity in response to chronic stress can cause cognitive impairment and reversible hippocampal atrophy (Brown et al. 2004; Miller and O'Callaghan 2005). In a longitudinal study of elderly people, those with elevated basal cortisol levels were more likely to have deficits in hippocampus-mediated declarative memory performance, as well as reduced hippocampal volumes (Lupien et al. 2005). One factor that may account for cognitive decline in subpopulations of the elderly is individual differences in the stress-response system, specifically genomic glucocorticoid signaling in the HPA axis (Goosens and Sapolsky 2007; Oitzl et al. 2009).

In the brain, the glucocorticoid receptor (GR) is ubiquitous and particularly enriched in the hippocampus, prefrontal cortex, hypothalamus, and amygdala in correspondence with their role in modulating HPA axis activity (Lupien et al. 2005). Results from a cellular system revealed an intracellular cascade involved in the mediation of GR in genomic glucocorticoid signaling in mammalian cells (Grad and Picard 2007; Wochnik et al. 2005). In the absence of ligand binding, the GR heterocomplex residing in the cytoplasm is assembled with molecular chaperones, mainly heat shock protein (Hsp) 90, p23, and immunophilin FK506 binding protein (FKBP) 51. Upon GR binding to its hormone ligands in the cytoplasm, FKBP51 (known to inhibit nuclear translocation of the GR heterocomplex by disrupting the attachment of the GR heterocomplex to the transport protein dynein) is competitively replaced by FKBP52 (Davies et al. 2002; Wochnik et al. 2005). By interacting with Hsp90, FKBP52, and dynein, GR is guided along cytoskeletal tracts to the nucleus to exert influences on specific gene transcription (Binder
2009). The activity of GR is therefore dependent on the relative levels of FKBP51 and FKBP52 in the cytoplasm (Grad and Picard 2007).

Examination of functional, anatomical, cellular, and neurochemical differences between aged animals that exhibit intact cognitive performance and those that are impaired has been pursued across rodents and nonhuman primates (Buccafusco 2006). In aged rats, elevated basal corticosterone levels predicted spatial memory impairment (Issa et al. 1990). In adult rodents, chronic stress or chronic exogenous administration of corticosterone caused reversible changes in the brain, such as dendritic retraction of pyramidal neurons in the prefrontal cortex and hippocampal CA3, decreased neurogenesis in the dentate gyrus, and hippocampal volume loss that was not associated with reduced neuron numbers (Lupien et al. 2009). It is not clear, however, whether downstream intracellular regulators of glucocorticoid signaling also play a role in cognitive decline.

To study the relationship between memory impairment and intracellular GR-mediated glucocorticoid signaling in aged mice, we assessed short-term recognition memory by using the single-trial object recognition test. This behavioral test is a relatively simple task of non-spatial recognition memory that does not require aversive learning (e.g., spatial navigation to escape aversive stimuli) or food restriction, which may alter baseline stress responses (Bevins and Besheer 2006; Dere et al. 2007). This task has been used in rodent models of aging among other studies (for review see Dere et al. 2007). We applied quantitative immunohistochemistry to assess cellular expression in the forebrain of three functionally related proteins in the GR-mediated glucocorticoid signaling (i.e., GR, FKBP51, and FKBP52) in aged mice compared to young mice. We hypothesized that alterations in GR signaling in the forebrain were associated with recognition memory impairment in aged mice.

\section{Materials and methods}

Behavioral testing

\section{Animals}

Young (6 months old, $n=23$ ) male C57BL/6N mice purchased from Charles River Laboratories (Wilmington, MA, USA), and aged (26 months old, $n=19$ ) mice from 
the National Institute of Aging stock located in Charles River Laboratories were used for single-trial object recognition testing. Mice were housed two per cage in a temperature-controlled room $\left(21-22^{\circ} \mathrm{C}\right)$ under a reverse 12/12-h light cycle. All procedures were in accordance with the "Principles of Laboratory Animal Care" (National Institute of Health [NIH] publication No. 86-23, revised 1985) and approved by the University of California, San Diego Animal Care Committee.

\section{Behavioral procedures}

The single-trial object recognition test was performed in an open arena $(60 \times 60 \mathrm{~cm})$ using two object types (i.e., Lego ${ }^{\mathrm{TM}}$ pyramid and 50-ml plastic conical tube) affixed to the floor with Velcro ${ }^{\mathrm{TM}}$ tape in opposing corners of the open field box, $10 \mathrm{~cm}$ away from the walls. Each mouse completed one session consisting of three successive trials. In Trial 1 , the habituation phase, the mouse was placed in the center of the empty open field box and allowed to freely explore. During Trial 2, the training phase, two identical objects of the same type were placed and the mouse was allowed to explore the objects. Half of the mice were randomly assigned to either starting with the Lego $^{\mathrm{TM}}$ pyramid or the conical tube as the familiar object. In Trial 3, the retention phase, one of the objects was replaced with a new object of the other type to assess novel object exploration. Each trial lasted for $5 \mathrm{~min}$ with a 3 -min inter-trial interval; these time periods were chosen to allow for the largest behavioral window to detect performance variance in aged mice. The mouse was removed from the testing box and placed in a holding cage during the inter-trial interval. The box and the objects were carefully cleaned with water between each trial and cleaned with $70 \%$ alcohol at the end of each testing session. Locomotor activity and object exploration were assessed using the Ethovision ${ }^{\mathrm{TM}}$ Tracking software (Noldus Information Technology, Wageningen, The Netherlands). Object exploration was scored using the Ethovision $^{\mathrm{TM}}$ Observe software when the mouse's nose was within $2 \mathrm{~cm}$ of the object.

\section{Behavioral analysis}

Overall locomotor activity was assessed by a one-way ANOVA with a factor of age across total distance, velocity, and zone entries during the habituation phase.
Object exploration during the retention phase was assessed by a two-way ANOVA with age as a between-subject factor and object (novel or familiar) as a within-subject factor. To normalize for exploration time, a one-way ANOVA with age as a between-subject factor was completed on the ratio of the time spent exploring the novel object over the total amount of time spent exploring both objects during the retention phase of the object recognition test (i.e., [novel object exploration time]/[novel object + familiar object exploration time]), referred to as the discrimination ratio (DR; Bevins and Besheer 2006). DR was used as a performance criterion for grouping aged mice as impaired aged or unimpaired aged. Aged mice with DR falling 1 standard deviation below the DR mean of the young mice $(n=23)$ group (i.e., below 0.54 , note that this DR was similar to the level of performance expected by chance) were classified as impaired ( $n=$ $10)$, and the remaining aged mice as unimpaired $(n=9)$. This statistical classification has been used in the literature to group cognitive performance across aged rodents in various cognitive tasks (Schoenbaum et al. 2006). The total amount of object exploration time was also assessed using a two-way ANOVA with age as a between-subject factor and phase (training vs. retention) as a within-subject factor. Data were initially analyzed with object and arena types as factors, but they did not interact with factors of age or performance group and thus were dropped from the analysis.

\section{Brain examination}

One of the impaired aged mice was found dead before sacrifice. Mice were sacrificed (decapitated) during 912 a.m. with order counterbalanced for age group to minimize the potential effect of circadian variation in the HPA axis activity. Brains were immediately dissected into two hemi-brains. One of the young mice was found to have a brain tumor and thus was excluded from the study. There were 18 aged mice (nine impaired and nine unimpaired) and 22 young mice available, the right hemi-brains of which were included in the immunohistologic study. The frontal lobe of one of the young mice was not available for the present study. All the right hemi-brains were immediately placed in $10 \%$ neutral buffered formalin and histologically processed for paraffin embedment in parasagittal plane. Fivemicrometer-thick tissue sections were taken with Superfrost/Plus microscope slides. One section from each 
hemi-brain was stained with hematoxylin and eosin for histologic examination.

\section{Immunohistochemistry for GR, FKBP52, and FKBP51}

Tissue sections were deparaffinized with xylene and rehydrated through graded ethanol series and water. Antigen retrieval was carried out at this step for FKBP51 with $10 \mathrm{mM}$ Tris/1 mM EDTA-2Na/0.05\% Tween 20 buffer (pH 9) in $121^{\circ} \mathrm{C}$ autoclave for $20 \mathrm{~min}$. The sections were treated for $30 \mathrm{~min}$ with $0.3 \%$ hydrogen peroxide in phosphate-buffered saline (PBS) to quench endogenous peroxidase activity, rinsed in PBS and incubated for 30 min with 5\% normal goat serum in PBS. Following $24 \mathrm{~h}$ incubation in humidified chambers at $4^{\circ} \mathrm{C}$ with rabbit polyclonal anti-GR (H-300, Santa Cruz Biotechnology, Santa Cruz, CA, USA, \#sc8992, 1:75 dilution in Dako antibody diluent, DakoCytomation, Carpinteria, CA, USA), anti-FKBP52 (ProteinTech Group, Chicago, IL, USA, \#10655-1AP, 1:100 dilution), or anti-FKBP51 (Abcam, Cambridge, MA, USA, \#ab2901, 1:300 dilution) primary antibody, the sections were rinsed in $0.1 \%$ Tween 20/ PBS and then incubated for $40 \mathrm{~min}$ in humidified chambers at room temperature with peroxidase-labeled polymer-conjugated goat anti-rabbit Ig secondary antibody (Envision+TM, DakoCytomation). Following washing with $0.1 \%$ Tween $20 / \mathrm{PBS}$, the signals were developed with diaminobenzidine (ImmPACT ${ }^{\mathrm{TM}} \mathrm{DAB}$ Peroxidase Substrate, Vector Laboratories, Burlingame, CA, USA) for 5 min. Following water wash, the sections were dehydrated through graded ethanol series, cleared in xylene and mounted with Cytoseal 60 (Richard-Allan Scientific, Waltham, MA, USA). For the negative reagent control, the primary antibody was omitted. For each of the three proteins studied, all the hemi-brains were processed in the same batch.

\section{Quantification of immunohistochemical reactivity}

The immunohistochemical reactivity was quantitatively assessed by means of two-dimensional computer-assisted image analysis. Briefly, the immunostained slides were imaged by using a Hitachi HVC20U-S4 3CCD color camera mounted on a Nikon Eclipse 80i microscope fitted with a Prior ProScan II motorized stage. With precise movement of the motorized stage controlled by the Stage-Pro module of the Image-Pro Plus version 4.5 software (Media Cybernetics, Bethesda, MD, USA) operating on Microsoft Windows XP, $640 \times 480$ pixel full-color image frames were contiguously captured within a scan area selected to cover the entire parasagittal brain section with a $\times 10$ objective lens and manual focus adjustment for each frame (the resolution being approximately $2 \mu \mathrm{m} / \mathrm{pixel}$ ). The Stage-Pro combined all the frames into a single-tiled image of the entire scan area without visible joints between frames, which was subsequently saved in Image-Pro Workspaces format. The same settings on the microscope (e.g., illumination, filters, and aperture), camera, and Image-Pro Plus for image acquisition were uniformly applied to all the hemi-brain sections studied.

On each image of the entire parasagittal hemi-brain section, the outline of the area of interest (AOI; i.e., the frontal cortex, dorsal hippocampal CA1, and granule cell layer of the dorsal dentate gyrus) was digitally drawn with the Image-Pro Plus software in accordance with the neuroanatomic landmarks (Valverde 1998; Fig. 1a-c, respectively). The first superficial layer of the frontal cortex was excluded to avoid staining artifacts, as were large vascular spaces and areas of tissue folding. To measure immunoreactive signals within AOI, histogram-based RGB color segmentation was set to best select the specific signal while ignoring the nonspecific background (Fig. 1d-f). For each pair of the three proteins and three neuroanatomic regions studied, the same setting of color segmentation was applied to all the hemi-brains. The values of three Image-Pro Plus measurement statistics including area (=area of object), integrated optical density (=[area] $\times$ [average optical density of object]) and per-area (=[area $] /[\mathrm{AOI}]$ ) were recorded for each AOI and exported to a Microsoft Excel spreadsheet. The value of AOI (in micrometer squared) was calculated by dividing the mean of area values by the mean of perarea values. The value of immunoreactivity normalized to the AOI (IRn) calculated by dividing the sum of integrated optical density values by the AOI value was used for subsequent statistical analysis.

Statistical analysis

Behavioral data from the single-trial object recognition test were in general not inconsistent with a Gaussian distribution ( $p>0.1$, Kolmogorov-Smirnov test) and showed no significant difference between the two 
Fig. 1 a-c Outlines of the frontal cortex $(F C)$, dorsal hippocampal CA1 (CA), and granule cell layer of the dorsal dentate gyrus $(D G)$, respectively, are digitally drawn (green) with the Image-Pro Plus version 4.5 software (Media Cybernetics, Bethesda, MD, USA) in accordance with the neuroanatomic landmarks (Valverde 1998). Immunoreactive signals (brown) for the glucocorticoid receptor $(G R)$ are shown within each of the three neuroanatomic regions. d-f With the Image-Pro Plus software, the histogram-based RGB color segmentation is set to best select the specific immunoreactive signals (red) while ignoring the nonspecific background. Note that right hemi-brain sections immunostained for GR and FK506 binding protein $(F K B P) 52$ are of one of the young mice, and that for FKBP51 is of one of the impaired aged mice (original magnification, $\times 100$ in a-f)
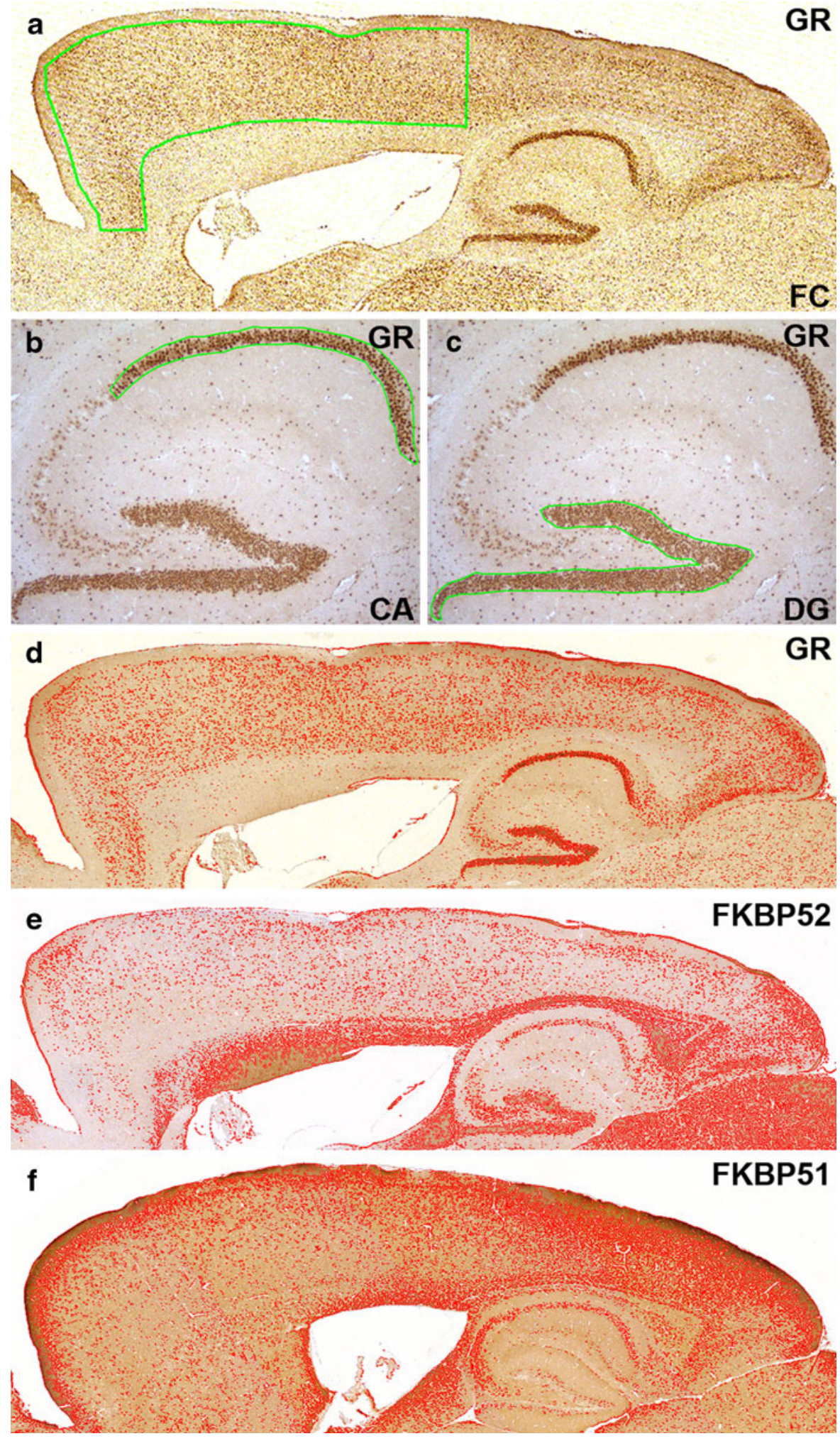
variances ( $p>0.2, F$ test) or among the three variances ( $p>0.6$, Bartlett's test); therefore, parametric methods were applied. In contrast, most of the immunohistochemical data were not normally distributed $(p<0.05$, Kolmogorov-Smirnov test) and nonparametric methods were employed accordingly. The Pearson productmoment correlation $(r)$ test and Spearman rank correlation $\left(r_{\mathrm{s}}\right)$ test were used to evaluate the linear relationship between two continuous variables in a given group. The unpaired Student $t$ test and MannWhitney $U$ test were employed to compare continuous variables between two independent groups. For three independent groups, we used the one-way ANOVA with the Tukey-Kramer post hoc test, and KruskalWallis test with the Dunn's post hoc test. The GraphPad InStat 3 for Macintosh software (GraphPad Software, La Jolla, CA, USA) was used to perform all statistical analysis. All $p$ values calculated were two-tailed and $p<0.05$ was considered statistically significant.

\section{Results}

Object recognition test

The aged $(n=18)$ and young $(n=22)$ mice groups did not differ in the total amount of time spent exploring both objects during the training phase (mean 24.98 vs. $21.24 \mathrm{~s}$, standard deviation [SD] 10.98 vs. $13.59 \mathrm{~s}$, $95 \%$ confidence interval $[95 \% \mathrm{CI}] 19.52-30.45$ vs. $15.22-27.27 \mathrm{~s}, p=0.352, t$ test). During the retention phase, aged mice spent significantly more time exploring both objects than young mice did (mean 30.79 vs. 19.73 s, SD 9.85 vs. 11.07 s, $95 \%$ CI $25.89-$ 35.69 vs. $14.82-24.64 \mathrm{~s}, p=0.002, t$ test), which was likely due to reduced habituation to the objects in aged mice. The DR distribution in aged mice was significantly lower than that in young mice (mean 0.54 vs. 0.67 , SD 0.10 vs. $0.14,95 \%$ CI $0.49-0.59$ vs. $0.61-$ $0.73, p=0.003, t$ test, Fig. 2). Compared to DR expected by chance (i.e., $50 \%$ exploration of the novel object), young mice exhibited significantly higher DR $(p<0.0001, t$ test), while aged mice did not $(p=0.081, t$ test). Nonetheless, DR of unimpaired aged mice significantly exceeded that would be expected by chance $(p<0.005, t$ test), while DR of impaired aged mice did not ( $p=0.102, t$ test). Four of the young mice exhibited DR lower than 0.54 . The impaired aged $(n=$

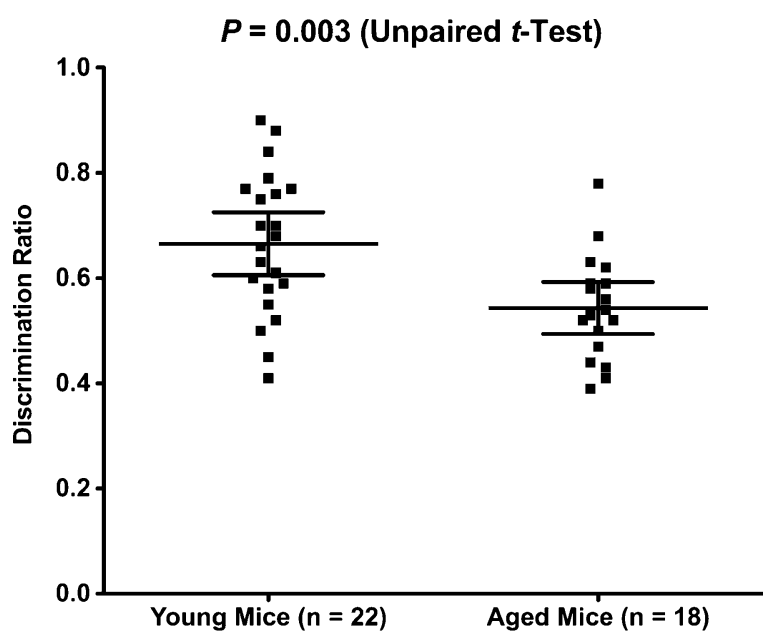

Fig. 2 The distribution of the discrimination ratio (i.e., the ratio of the time spent exploring the novel object over the total amount of time spent exploring both familiar and novel objects during the retention phase of the single-trial object recognition test) in aged mice is significantly lower than that in young mice (mean 0.54 vs. 0.67 , standard deviation 0.10 vs. $0.14,95 \%$ confidence interval $0.49-0.59$ vs. $0.61-0.73$ ). The long horizontal line and error bar in each group of mice represent the mean value and $95 \%$ confidence interval, respectively. The $p$ value is two-tailed

9), unimpaired aged ( $n=9$ ), and young $(n=22)$ mice groups did not differ in the total amount of time spent exploring both objects during the training phase (mean 25.25 vs. 24.71 vs. 21.24 s, SD 10.06 vs. 12.45 vs. 13.59 s, $95 \%$ CI $17.52-32.99$ vs. $15.14-34.28$ vs. 15.22-27.27 s, $p=0.65$, one-way ANOVA). During the retention phase, there was a significant difference between the impaired aged, unimpaired aged, and young mice groups in the total amount of time spent exploring both objects (mean 31.65 vs. 29.92 vs. 19.73 s, SD 8.88 vs. 11.21 vs. 11.07 s, $95 \%$ CI $24.83-$ 38.48 vs. $21.31-38.54$ vs. $14.82-24.64 \mathrm{~s}, p=0.009$, one-way ANOVA), but no significant difference was observed between the impaired and unimpaired aged mice groups in this regard ( $p>0.05$, Tukey-Kramer post hoc test). In aged mice, there was no significant correlation between DR and the total amount of time spent exploring both objects during either the training or retention phase $(r=-0.23$ and $-0.38, p=0.36$ and 0.12 , respectively, Pearson correlation, $n=18$ ).

Histologic examination

All the parasagittal hemi-brain sections revealed neuroanatomic landmarks consistent with the levels 
from 750 to $1,250 \mu \mathrm{m}$ from the midline (Valverde 1998). No significant histopathologic changes were observed in any sections.

Quantitative immunohistochemistry

The IRn values for GR, FKBP52, and FKBP51 in the frontal cortex, dorsal hippocampal CA1, and granule cell layer of the dorsal dentate gyrus are shown as the median and interquartile range in Table 1.

$G R$

Immunoreactive signals for GR (Fig. 3a-c) were observed in neuronal nuclei (weak to intense) and cytoplasm (weak) in the cerebral cortex (Fig. 3a), hippocampal CA1 (intense in nuclei; Fig. 3b) and CA3 (weak in nuclei), granule cell layer of the dentate gyrus (intense in nuclei; Fig. 3c), olfactory bulb, caudate-putamen, thalamus (intense in nuclei and weak in cytoplasm; Fig. 3c, inset), hypothalamus, midbrain, brainstem, and cerebellum (in Purkinje cells, granule cells, and some neurons in the molecular layer). Oligodendroglial nuclei were also moderately to intensely immunoreactive.

The distribution of GR IRn (Fig. 4) was significantly different across the three groups of mice in all the three regions studied $(p=0.017$ [frontal cortex], $<0.001$ [CA1], and $=0.001$ [dentate gyrus], Kruskal-Wallis test). No significant difference in the GR IRn was present between impaired and unimpaired aged mice in any of the three regions studied ( $p>0.05$, Dunn's post hoc test). The GR IRn was significantly increased in aged mice compared to young mice in all the three regions studied, i.e., the frontal cortex $(p=0.004, U$ test), CA1 $(p<0.001, U$ test), and dentate gyrus $(p<$ $0.001, U$ test).

\section{FKBP52}

Immunoreactive signals for FKBP52 were shown in neuronal cytoplasm (weak to intense) and nuclei (weak) in the cerebral cortex (Fig. 3d); hippocampal CA1, 2, and 3 (Fig. 3e, f); granule cell layer of the dentate gyrus (Fig. 3e, g); olfactory bulb; caudateputamen; posterior part of the thalamus (Fig. 3g, inset); hypothalamus; midbrain; brainstem; and cerebellum (in granule cells and neurons in the molecular layer). Within individual neurons, the immunoreactivity appeared stronger in the cytoplasm compared to that in the nucleus (Fig. 3g, inset). The white matter tracts were moderately to intensely immunoreactive.

The distribution of FKBP52 IRn (Fig. 4) was significantly different across the three groups of mice in all the three regions studied $(p<0.001$ [frontal cortex], $=0.015$ [CA1], and $=0.003$ [dentate gyrus]; Kruskal-Wallis test). No significant difference in the FKBP52 IRn was present between impaired and

Table 1 Median (interquartile range) values of the immunoreactivity normalized to the neuroanatomic area measured (IRn)

\begin{tabular}{lcccc}
\hline & Young mice $(n=22)^{\mathrm{a}}$ & Aged mice $(n=18)$ & Unimpaired aged mice $(n=9)$ & Impaired aged mice $(n=9)$ \\
\hline GR IRn & & & \\
FC & $5.54(3.96)$ & $9.12(5.83)$ & $9.17(6.05)$ & $9.06(4.65)$ \\
CA & $45.76(9.69)$ & $59.46(7.48)$ & $59.32(7.59)$ & $60.25(7.42)$ \\
DG & $36.87(24.30)$ & $58.16(13.82)$ & $60.09(15.55)$ & $57.98(10.39)$ \\
FKBP52 IRn & & & & $5.85(4.10)$ \\
FC & $1.71(2.75)$ & $5.65(2.95)$ & $10.54(3.87)$ & $13.88(7.08)$ \\
CA & $3.18(8.68)$ & $10.98(6.45)$ & $33.55(17.63)$ & $28.46(11.22)$ \\
DG & $12.35(14.98)$ & $30.52(17.21)$ & & $8.47(1.93)$ \\
FKBP51 IRn & & $14.66(17.76)$ & $27.85(23.01)$ & $33.65(14.38)$ \\
FC & $19.68(13.72)$ & $44.03(30.21)$ & $61.02(17.32)$ & $17.17(8.26)$ \\
CA & $58.30(21.12)$ & $19.11(9.92)$ & $22.03(11.10)$ & \\
DG & $27.62(16.76)$ & & \\
\hline
\end{tabular}

$G R$ glucocorticoid receptor, $F K B P$ FK506 binding protein, $F C$ frontal cortex, $C A$ dorsal hippocampal CA1, $D G$ granule cell layer of the dorsal dentate gyrus

${ }^{\mathrm{a}}$ The number of mice $(n)$ is 21 for $\mathrm{FC}$ 

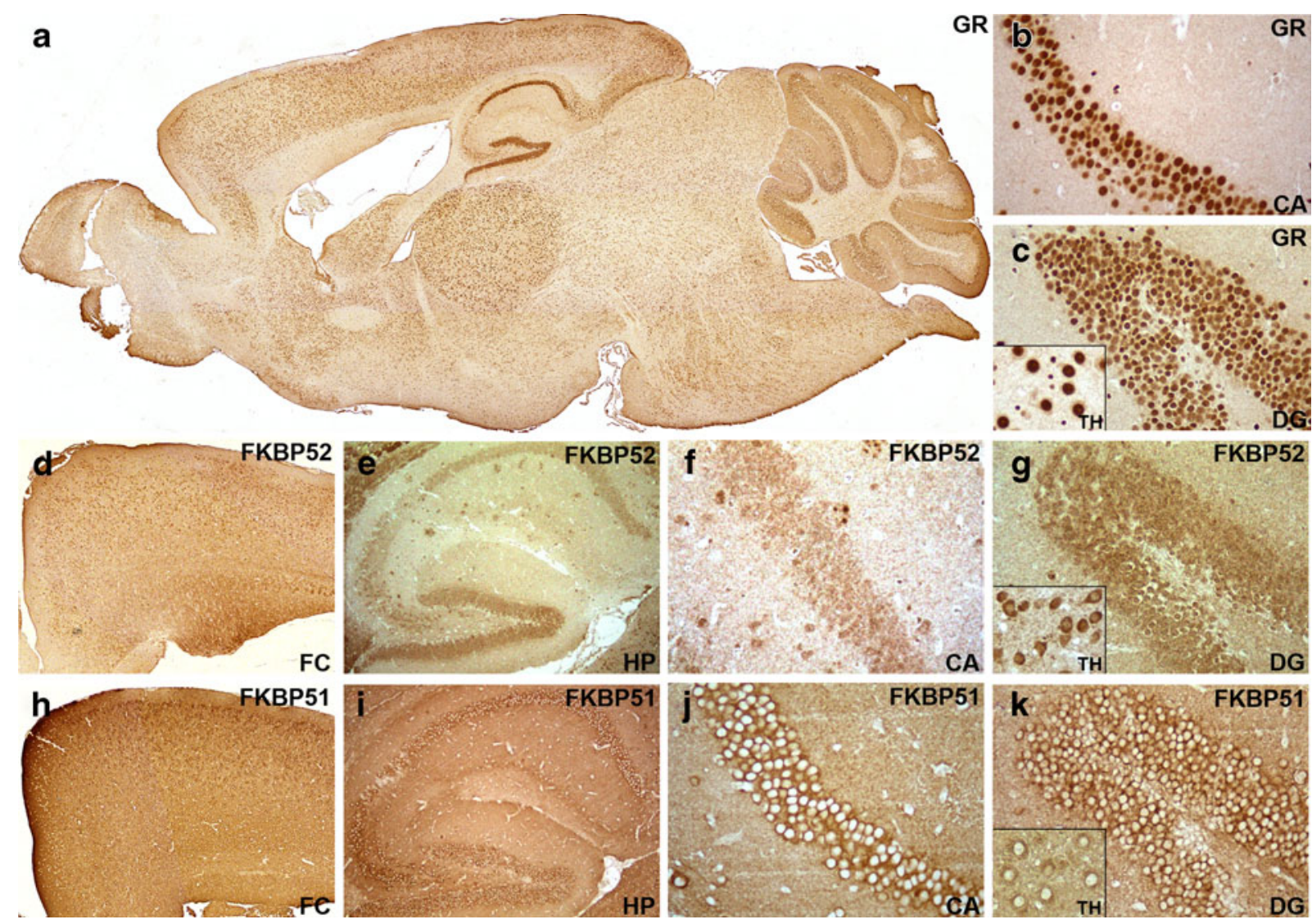

Fig. 3 a The distribution of glucocorticoid receptor $(G R)$ formation $[H P], \mathrm{CA}, \mathrm{DG}$, and $\mathrm{TH}[\mathrm{g}$ inset]) and nuclei (weak; in $\mathrm{TH}$ [g inset]). h-k Immunoreactive signals for FKBP51 are observed in neuronal cytoplasm (weak to intense; in FC, HP, $\mathrm{CA}, \mathrm{DG}$, and TH [k inset $]$ ) but not in neuronal nuclei. Note that right hemi-brain sections immunostained for GR and FKBP51 are of one of the young mice, and that for FKBP52 is of one of the unimpaired aged mice (original magnification, $\times 100$ in $\mathbf{a}, \mathbf{d}$, $\mathbf{e}, \mathbf{h}$, and $\mathbf{i}$ and $\times 400$ in $\mathbf{b}, \mathbf{c}, \mathbf{f}, \mathbf{g}, \mathbf{j}$, and $\mathbf{k}$ ) $(F K B P) 52$ are observed in neuronal cytoplasm (weak to intense; in the frontal cortex $[F C]$, dorsal hippocampal

unimpaired aged mice in any of the three regions studied ( $p>0.05$, Dunn's post hoc test). The FKBP52 IRn was significantly increased in aged mice compared to young mice in all the three regions studied, i.e., the frontal cortex $(p<0.001, U$ test), CA1 ( $p=$ 0.006, $U$ test), and dentate gyrus ( $p=0.001, U$ test $)$.

\section{FKBP51}

Weak to intense immunoreactive signals for FKBP51 were present in neuronal cytoplasm in the cerebral cortex (Fig. 3h); hippocampal CA1, 2, and 3 (Fig. 3i, j); granule cell layer of the dentate gyrus (Fig. 3i, k); olfactory bulb; caudate-putamen; thalamus (Fig. 3k,

inset); hypothalamus; midbrain; brainstem; and cerebellum (in granule cells). No immunoreactive signals were observed in neuronal nuclei. The white matter tracts were weakly to moderately immunoreactive.

The distribution of FKBP51 IRn (Fig. 4) was significantly different across the three groups of mice in all the three regions studied $(p=0.003$ [frontal cortex], $=0.002$ [CA1], and $=0.04$ [dentate gyrus]; Kruskal-Wallis test). In the frontal cortex, the FKBP51 IRn was significantly lower in impaired aged mice than in unimpaired aged mice $(p<0.01$, Dunn's post hoc test) and in young mice ( $p<0.05$, Dunn's post hoc test). Also in the CA1, the FKBP51 IRn was significantly lower in impaired aged mice than in unimpaired aged 
口Young aUnimpaired Aged almpaired Aged

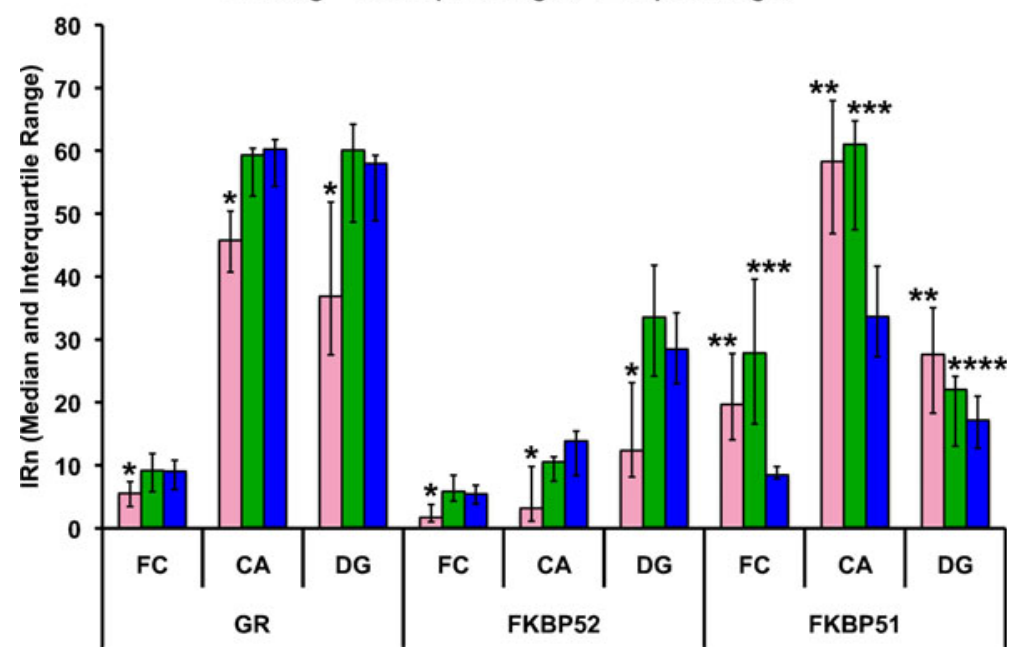

Fig. 4 Median values of the immunoreactivity normalized to the neuroanatomic area measured $(I R n)$ in the frontal cortex $(F C)$, dorsal hippocampal CA1 $(C A)$, and granule cell layer of the dorsal dentate gyrus $(D G)$ for the glucocorticoid receptor $(G R)$, FK506 binding protein $(F K B P)$ 52, and FKBP51 are shown. The error bars represent the interquartile ranges. Note that under the same setting of color segmentation for computer-assisted quantification of immunoreactive signals, the IRn values for each of the three protein markers in each of the three neuroanatomic regions are compared among three groups of mice (i.e., young $[n=21$ for FC, $n=22$ for CA and DG], unimpaired aged $[n=9]$, and impaired aged [ $n=9]$ groups), classified based on age and performance on the single-trial object recognition test. The IRn values cannot be precisely

mice ( $p<0.05$, Dunn's post hoc test) and in young mice $(p<0.01$, Dunn's post hoc test). In the dentate gyrus, the FKBP51 IRn was significantly decreased in impaired aged mice compared to young mice $(p<$ 0.05 , Dunn's post hoc test); nonetheless, the difference in the FKBP51 IRn between impaired and unimpaired aged mice did not reach statistical significance $(p>$ 0.05, Dunn's post hoc test). The FKBP51 IRn in unimpaired aged mice was not significantly different from that in young mice in any of the three regions studied ( $p>0.05$, Dunn's post hoc test).

In aged mice, the FKBP51 IRn in the frontal cortex (Fig. 5) and CA1 showed significant direct correlation with DR $\left(r_{\mathrm{s}}=0.68\right.$ and $0.53, p=0.002$ and 0.024 , respectively, Spearman rank correlation). In the dentate gyrus of aged mice, correlation between the FKBP51 IRn and DR did not reach statistical significance $\left(r_{\mathrm{s}}=\right.$ $0.28, p=0.26$, Spearman rank correlation). In young mice, no significant correlation was present between the FKBP51 IRn and DR in any of the three regions compared among different protein markers or different neuroanatomic regions. * The IRn values for GR and FKBP52 in all the three neuroanatomic regions are significantly higher in aged mice than those in young mice ( $p<0.007$, two-tailed Mann-Whitney $U$ test; see text for individual exact $p$ values). ${ }^{* *}$ The FKBP51 IRn values in FC, CA, and DG are significantly lower in impaired aged mice than those in young mice $(p<0.05,<0.01$, and $<0.05$, respectively, two-tailed Dunn's post hoc test). *** The FKBP51 IRn values in FC and CA are significantly lower in impaired aged mice than those in unimpaired aged mice $(p<0.01$ and $<0.05$, respectively, two-tailed Dunn's post hoc test). ${ }^{* * * *}$ In DG, the difference in the FKBP51 IRn between impaired and unimpaired aged mice does not reach statistical significance $(p>$ 0.05, two-tailed Dunn's post hoc test)

studied, i.e., the frontal cortex $\left(r_{\mathrm{s}}=-0.16, p=0.49\right)$, CA1 $\left(r_{\mathrm{s}}=0.02, p=0.93\right)$, and dentate gyrus $\left(r_{\mathrm{s}}=0.02\right.$, $p=0.99$, Spearman rank correlation).

With regard to the time spent by aged mice exploring both objects during the retention phase, no significant correlation was observed with the FKBP51 IRn in any of the three regions studied, i.e., the frontal cortex $\left(r_{\mathrm{s}}=-0.15, p=0.56\right)$, CA1 $\left(r_{\mathrm{s}}=0.23, p=0.36\right)$, and dentate gyrus $\left(r_{\mathrm{s}}=0.27, p=0.28\right.$, Spearman rank correlation), nor was it present during the training phase $\left(r_{\mathrm{s}}=0.19,0.1\right.$, and $0.35, p=0.46,0.68$ and 0.15 , respectively, Spearman rank correlation).

\section{FKBP51/FKBP52 ratios}

Because FKBP51 was shown in previous studies to act as an inhibitor and FKBP52 as a facilitator for GR translocation to the nucleus (Grad and Picard, 2007; Wochnik et al., 2005), we explored the relationship between the object recognition performance and the 
ratio of FKBP51 IRn to FKBP52 IRn. The FKBP51/ FKBP52 ratios were significantly different across the three groups of mice in all the three regions studied $(p<0.0001$ [frontal cortex], $=0.0004$ [CA1], and $=0.0003$ [dentate gyrus]; Kruskal-Wallis test). In the frontal cortex, CA1, and dentate gyrus, the FKBP51/ FKBP52 ratios were significantly lower in impaired aged mice than in young mice $(p<0.001,<0.001$, and $<0.01$, respectively, Dunn's post hoc test).

In young mice, the FKBP51/FKBP52 ratio exhibited significant direct correlation in each pair of the three regions studied, i.e., the frontal cortex vs. CA1, frontal cortex vs. dentate gyrus, and CA 1 vs. dentate gyrus $\left(r_{\mathrm{s}}=\right.$ $0.77,0.59$, and $0.76, p<0.0001,=0.005$, and $<0.0001$, respectively, Spearman rank correlation). In aged mice, the significant direct correlation was observed in the frontal cortex vs. CA1 $\left(r_{\mathrm{s}}=0.6, p=0.009\right)$ and in the CA1 vs. dentate gyrus $\left(r_{\mathrm{s}}=0.5, p=0.03\right)$, but not in the frontal cortex vs. dentate gyrus $\left(r_{\mathrm{s}}=0.28, p=0.26\right)$.

In aged mice, the FKBP51/FKBP52 ratio in the frontal cortex and CA1 showed significant direct correlation with DR $\left(r_{\mathrm{s}}=0.60\right.$ and $0.69, p=0.009$ and $=0.002$, respectively, Spearman rank correlation). In the dentate gyrus of aged mice, correlation between the FKBP51/FKBP52 ratio and DR did not reach statistical significance $\left(r_{\mathrm{s}}=0.25, p=0.31\right.$, Spearman rank correlation).

\section{Discussion}

A large proportion of the elderly are affected with a variable degree of cognitive decline that is not attributable to a defined clinical entity (Goosens and Sapolsky 2007; Lupien et al. 2005). It is of particular interest to study the differences at cellular and neurochemical levels in the brain between successful and non-successful elderly individuals with regard to cognitive aging. By using experimental animals, we applied the single-trial object recognition test to assess short-term recognition memory in aged mice compared to young mice, and quantitative immunohistochemistry to measure cellular expression of three proteins involved in intracellular genomic glucocorticoid signaling (i.e., GR, FKBP51, and FKBP52) in the brain. GR is ubiquitously distributed in neurons and glial cells (de Kloet et al. 2005; Joëls 2001). The patterns of cellular immunoreactivity for GR (both in the nucleus and cytoplasm), FKBP52 (both in the cytoplasm and nucleus), and FKBP51 (only in the cytoplasm) shown in the present study were consistent with a proposed mechanism of the GR heterocomplex trafficking from the cytoplasm to the nucleus, i.e., upon GR binding to glucocorticoids, FKBP51 in the GR heterocomplex is competitively replaced by FKBP52 to facilitate translocation to the nucleus (Grad and Picard 2007; Wochnik et al. 2005).

We found in our present study that GR and FKBP52 expression were increased in aged mice irrespective of their object recognition performance compared to young mice, while FKBP51 expression was decreased only in aged mice with recognition memory impairment. In mammalian cells, the activity of GR is dependent on the relative levels of FKBP51 and FKBP52 in the cytoplasm where FKBP51 acts as an inhibitor and FKBP52 as a facilitator for nuclear translocation of GR upon hormone binding (Grad and Picard 2007; Wochnik et al. 2005). Our data further showed that the FKBP51/FKBP52 ratio correlated directly with DR in aged mice. These findings suggest that GR-mediated glucocorticoid signaling in the forebrain is increased with age by up-regulation of GR and FKBP52, and aging-related impairment in short-term recognition memory is associated with the concomitant FKBP51 down-regulation that further promotes glucocorticoid signaling. In agreement with our findings, a study using transgenic mice suggested that GR over-expression in forebrain was associated with accelerated aging-related deficiencies in feedback inhibition of the HPA axis activity, as well as mild dysfunction in long-term spatial memory, which was observed after reversal learning training in the Morris water maze test (Wei et al. 2007).

The single-trial object recognition test for rodents is a novelty-preference paradigm aimed at assessing non-spatial recognition memory and requiring innate motivation to explore novel stimuli (Bevins and Besheer 2006; Dere et al. 2007). This behavioral test measures the ability to recognize a familiar object and discriminate it from a novel object (Dere et al. 2007). Because aged mice spent significantly more time exploring both objects than young mice did during the retention phase, we tested whether there was any relationship between the FKBP51 expression and the total object exploration time. It appeared that the findings of FKBP51 expression were relatively selective to the object memory performance, as this neurochemical marker was not significantly correlated 
with the total object exploration time during either the training or retention phase in aged mice. Moreover, it does not appear that the direct correlation between the FKBP51 expression and DR in aged mice (Fig. 5) can be explained by differences in exploration or motivation. The total object exploration time during the retention phase did not predict DR in aged mice, nor did it correlate with the FKBP51 expression, suggesting this exploration time measure is orthogonal to DR. It is possible that aged animals showed reduced habituation to the objects compared to young animals. Dysregulation in GR signaling in the forebrain may be one of several potential mechanisms involved in aging-related impairment in short-term recognition memory. Any genomic GR signaling is not likely to occur during the behavioral task of relatively short period in the single-trial object recognition test. The combination of decreased FKBP51 expression and increased expression of GR and FKBP52 in forebrain in our present study may represent the molecular substrate for exaggerated genomic glucocorticoid signaling that adversely affects the functional condition of mice prior to the learning task and consequently predisposes them to develop short-term recognition memory impairment.

The GR-mediated effects of glucocorticoids are implicated in terminating ongoing stress reaction, facil-

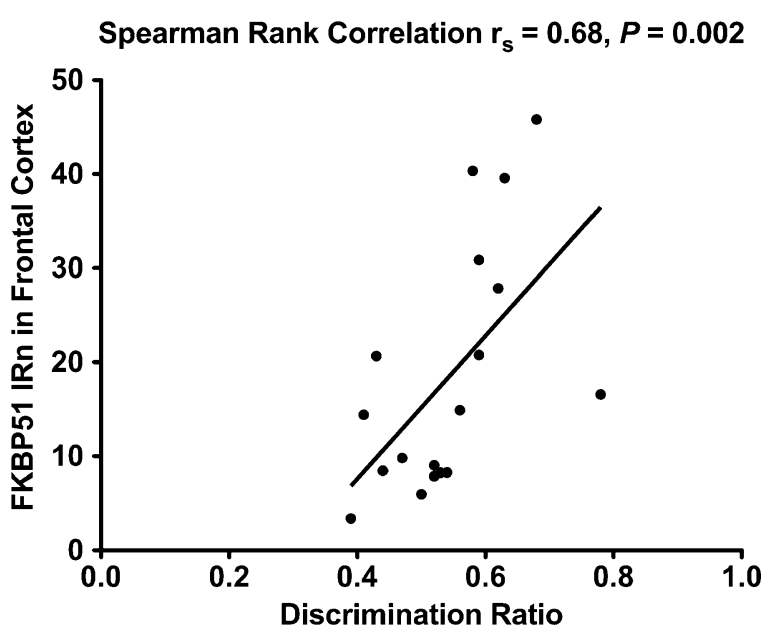

Fig. 5 Scattergraph with its trend line shows significant direct correlation between the immunoreactivity normalized to the neuroanatomic area measured $(I R n)$ in the frontal cortex of aged mice $(n=18)$ for the FK506 binding protein $(F K B P) 51$ and the discrimination ratio (i.e., the ratio of the time spent exploring the novel object over the total amount of time spent exploring both familiar and novel objects during the retention phase of the single-trial object recognition test). The $p$ value is two-tailed itating recovery, and promoting memory storage in preparation for future events (de Kloet et al. 2005). Upon GR activation, increased influx of calcium through voltage-gated calcium channels helps to slowly reverse temporarily raised electrical activity in adaptive response to stress (de Kloet et al. 2005; Joëls 2001). Aging-related up-regulation of GR and FKBP52 found in our present study may form the molecular basis for excessive GR-mediated glucocorticoid signaling during stress response. In this maladaptive reaction, calcium influx may be enhanced beyond control, predisposing neurons in vulnerable brain regions to injury (de Kloet et al. 2005; Joëls 2001). Moreover, the concomitant down-regulation of FKBP51 observed in our impaired aged mice might represent the molecular substrate for exaggerated GR-mediated glucocorticoid signaling during stress reaction. The potential neurobiological correlates of aging-related cognitive impairments include dendritic atrophy, synaptic degeneration, and deficiency in adult hippocampal neurogenesis (McEwen and Magarinos 2001; Miller and O'Callaghan 2005). Experimental studies in mice showed that overall levels of adult neurogenesis drastically declined in old age, although voluntary physical exercise and long-term environmental enrichment could induce neurogenesis (Kempermann et al. 2002; McEwen and Magarinos 2001; van Praag et al. 1999). In the subgranular layer of the dentate gyrus, GR expression in neural precursor cells involved in adult neurogenesis was shown to be increased in aged mice compared to young mice, which might enhance their vulnerability to adverse glucocorticoid-mediated influences (Garcia et al. 2004).

There are individual differences in basal and stressresponse levels of circulating glucocorticoids, depending on genetic processes or epigenetic modifications associated with early-life psychosocial experiences affecting the HPA axis function (Goosens and Sapolsky 2007). Aging-related changes in basal corticosterone levels in rodents might be strain dependent (Herbert et al. 2006). In the rat, elevated corticosterone levels were not a necessary consequence of aging, but were more prevalent in those exhibiting spatial memory deficits than in cognitively intact animals (Herbert et al. 2006). By using the Morris water maze test to assess hippocampus-dependent spatial learning, Bizon et al. (2001) found no differences in basal or peakstress-induced levels of circulating corticosterone between young or aged rats, irrespective of cognitive status. However, plasma corticosterone levels in 
cognitively impaired aged rats were slower to return to baseline after acute restraint stress compared to young or unimpaired aged rats. By in situ hybridization, GR mRNA levels were lower in the ventral hippocampal CA1 and CA3, dorsal and ventral dentate granule cell layer, and medial prefrontal cortex of impaired aged rats compared to young rats; no quantitative GR protein data shown in this report (Bizon et al. 2001). In a study using transgenic mice with forebrain-specific GR over-expression, Wei et al. (2007) reported that both young and aged transgenic mice in response to strong acute restraint stress displayed a delayed termination of circulating corticosterone elevation, compared to the respective wild-type mice. Specifically, following strong acute stress young transgenic mice exhibited a corticosterone recovery pattern similar to that seen in aged wild-type mice. This finding suggested that forebrain-dependent feedback inhibition of the HPA axis activity was deficient in GRoverexpressed mice, which might be related to decreased gene expression in the glutamate receptor signaling system shown in the RNA microarray analysis of the hippocampus (Wei et al. 2007). In our present study, circulating levels of corticosterone were not measured in relation to object recognition performance or stress response, nor were GR mRNA expression levels in the forebrain.

In addition to the role in GR signaling, FKBP52 with its ability to bind Hsp90 is involved in the intracellular signaling of progesterone, estrogen, and androgen receptors (Davies and Sánchez 2005). Most of FKBP immunophilin family members (e.g., FKBP12, FKBP51, and FKBP52) exhibit peptidyl-prolyl cistrans isomerase activity, which is involved in the slow protein-folding process (Kang et al. 2008). Unlike FKBP12, FKBP52 when bound to the immunosuppressive drug FK506 does not inhibit calcineurin involved in T cell activation (Davies and Sánchez 2005). FKBP38 can protect cells from apoptosis by localizing anti-apoptotic proteins $\mathrm{Bcl}-2$ and $\mathrm{Bcl}-\mathrm{X}_{\mathrm{L}}$ to the mitochondrial membrane (Kang et al. 2008). Immunophilin ligands (e.g., FK506, cyclosporin A, and rapamycin) have been reported to have neuroprotective effects via unclear mechanisms (Kang et al. 2008). Nonimmunosuppressive rapamycin analogs, WYE-592 and ILS-920, were shown to protect neurons from calcium-induced cell death by modulating calcium channels and promote neuronal survival and neurite outgrowth through FKBP52 binding (Ruan et al. 2008).
In summary, our present study correlated the combination of decreased FKBP51 expression and increased expression of GR and FKBP52 in forebrain with short-term recognition memory impairment in aged mice. This intracellular neurochemical dysregulation may represent the molecular substrate for exaggerated GR-mediated glucocorticoid signaling during stress response. Extensive evidence has suggested that chronic stress and prolonged elevation of circulating glucocorticoids of various etiologies have deleterious effects on cognitive function (Brown et al. 2004; Goosens and Sapolsky 2007; Miller and O'Callaghan 2005). Glucocorticoids, while their circulating levels are high, need to first bind to GR in the cytoplasm in order to initiate a cascade of nuclear translocation of the GR heterocomplex to eventually exert their genomic influences on target cells either in adaptive or maladaptive direction (Grad and Picard 2007; Wochnik et al. 2005). These GR-dependent effects may be complemented by faster non-genomic effects mediated by the membrane mineralocorticoid receptor (MR) displaying a 10-fold lower affinity for corticosterone than nuclear MR (de Kloet et al. 2008). Even in the absence of chronic stress or prolonged excessive levels of circulating glucocorticoids, the combination of decreased FKBP51 expression and increased expression of GR and FKBP52 in the brain may lead to abnormal glucocorticoid signaling particularly in response to sporadic acute stress.

Excessive glucocorticoid signaling may accelerate neural damage such as dendritic atrophy and synaptic degeneration (McEwen and Magarinos 2001; Miller and O'Callaghan 2005). On the other hand, the concurrence of high circulating levels of glucocorticoids and GR-mediated glucocorticoid resistance in target cells may less likely contribute to tissue damage, as seen in New World monkeys having hypercortisolemia without clinical signs of hypercortisolism (Binder 2009; Westberry et al. 2006). In the present study, we did not directly assess levels of GR signaling in the brain by treating mice with glucocorticoids and then measuring transcriptional levels of cellular responses; nonetheless, this experimental approach can be conducted in future studies. In addition to the HPA axis activity, studies of other potential mechanisms that underlie aging-related cognitive decline, such as oxidative stress and neuroinflammation, can be pursued. Oxidative modifications affect virtually all classes of cellular macromolecules. Oxidative markers 
like 8-hydroxyguanosine and 3-nitrotyrosine can be studied on tissue sections by quantitative immunohistochemistry to assess RNA and protein oxidation, respectively (Nunomura et al. 2009). We assessed short-term recognition memory in aged mice by using the single-trial object recognition test; thus, our findings may not be generalized to other domains of cognitive impairment.

Acknowledgments This work was supported in part by a Sam and Rose Stein Institute for Research on Aging fellowship (J. W. Young), a junior faculty pilot award (V. B. Risbrough), the Veterans Affairs Center of Excellence for Stress and Mental Health (V. B. Risbrough), the United States National Institute of Health (NIH) grant MH052885 (V. B. Risbrough), an interdisciplinary research fellowship in neuroAIDS (V. Soontornniyomkij) in the NIH grant MH81482, and the NIH grant MH76681 (C. L. Achim).

Disclosure The authors declare that they have no conflict of interest.

Open Access This article is distributed under the terms of the Creative Commons Attribution Noncommercial License which permits any noncommercial use, distribution, and reproduction in any medium, provided the original author(s) and source are credited.

\section{References}

Bevins RA, Besheer J (2006) Object recognition in rats and mice: a one-trial non-matching-to-sample learning task to study 'recognition memory'. Nat Protoc 1:1306-1311

Binder EB (2009) The role of FKBP5, a co-chaperone of the glucocorticoid receptor in the pathogenesis and therapy of affective and anxiety disorders. Psychoneuroendocrinology 34(Suppl 1):S186-S195

Bizon JL, Helm KA, Han J-S, Chun H-J, Pucilowska J, Lund PK, Gallagher M (2001) Hypothalamic-pituitary-adrenal axis function and corticosterone receptor expression in behaviourally characterized young and aged Long-Evans rats. Eur J NeuroSci 14:1739-1751

Brown ES, J Woolston D, Frol A et al (2004) Hippocampal volume, spectroscopy, cognition, and mood in patients receiving corticosteroid therapy. Biol Psychiatry 55:538-545

Buccafusco JJ (2006) Cognitive pharmacology in aging macaques. In: Levin ED, Buccafusco JJ (eds) Animal models of cognitive impairment. CRC, Boca Raton, pp 285-287

Davies TH, Sánchez ER (2005) FKBP52. Int J Biochem Cell Biol 37:42-47

Davies TH, Ning YM, Sánchez ER (2002) A new first step in activation of steroid receptors: hormone-induced switching of FKBP51 and FKBP52 immunophilins. J Biol Chem 277:4597-4600

de Kloet ER, Joëls M, Holsboer F (2005) Stress and the brain: from adaptation to disease. Nat Rev Neurosci 6:463-475 de Kloet ER, Karst H, Joëls M (2008) Corticosteroid hormones in the central stress response: quick-and-slow. Front Neuroendocrinol 29:268-272

Depp CA, Glatt SJ, Jeste DV (2007) Recent advances in research on successful or healthy aging. Curr Psychiatry Rep 9:7-13

Dere E, Huston JP, De Souza Silva MA (2007) The pharmacology, neuroanatomy and neurogenetics of onetrial object recognition in rodents. Neurosci Biobehav Rev 31:673-704

Garcia A, Steiner B, Kronenberg G, Bick-Sander A, Kempermann G (2004) Age-dependent expression of glucocorticoid- and mineralocorticoid receptors on neural precursor cell populations in the adult murine hippocampus. Aging Cell 3:363-371

Goosens KA, Sapolsky RM (2007) Stress and glucocorticoid contributions to normal and pathological aging. In: Riddle DR (ed) Brain aging: models, methods, and mechanisms. CRC, New York, pp 305-314

Grad I, Picard D (2007) The glucocorticoid responses are shaped by molecular chaperones. Mol Cell Endocrinol 275:2-12

Herbert J, Goodyer IM, Grossman AB et al (2006) Do corticosteroids damage the brain? J Neuroendocrinol 18:393-411

Issa AM, Rowe W, Gauthier S, Meaney MJ (1990) Hypothalamic-pituitary-adrenal activity in aged, cognitively impaired and cognitively unimpaired rats. J Neurosci $10: 3247-3254$

Joëls M (2001) Corticosteroid actions in the hippocampus. J Neuroendocrinol 13:657-669

Kang CB, Hong Y, Dhe-Paganon S, Yoon HS (2008) FKBP family proteins: immunophilins with versatile biological functions. Neurosignals 16:318-325

Kempermann G, Gast D, Gage FH (2002) Neuroplasticity in old age: sustained fivefold induction of hippocampal neurogenesis by long-term environmental enrichment. Ann Neurol 52:135-143

Lupien SJ, Fiocco A, Wan N, Maheu F, Lord C, Schramek T, Tu MT (2005) Stress hormones and human memory function across the lifespan. Psychoneuroendocrinology 30:225-242

Lupien SJ, McEwen BS, Gunnar MR, Heim C (2009) Effects of stress throughout the lifespan on the brain, behaviour and cognition. Nat Rev Neurosci 10:434-445

McEwen BS, Magarinos AM (2001) Stress and hippocampal plasticity: implications for the pathophysiology of affective disorders. Hum Psychopharmacol 16:S7-S19

Miller DB, O'Callaghan JP (2005) Aging, stress and the hippocampus. Ageing Res Rev 4:123-140

Nunomura A, Hofer T, Moreira PI, Castellani RJ, Smith MA, Perry G (2009) RNA oxidation in Alzheimer disease and related neurodegenerative disorders. Acta Neuropathol 118:151-166

Oitzl MS, Champagne DL, van der Veen R, de Kloet ER (2009) Brain development under stress: hypotheses of glucocorticoid actions revisited. Neurosci Biobehav Rev. doi:10.1016/j.neubiorev.2009.07.006

Ruan B, Pong K, Jow F et al (2008) Binding of rapamycin analogs to calcium channels and FKBP52 contributes to their neuroprotective activities. Proc Natl Acad Sci U S A 105:33-38 
Schoenbaum G, Setlow B, Saddoris MP, Gallagher M (2006) Encoding changes in orbitofrontal cortex in reversalimpaired aged rats. J Neurophysiol 95:1509-1517

Valverde F (1998) Golgi atlas of the postnatal mouse brain. Springer/Wein, New York

van Praag H, Kempermann G, Gage FH (1999) Running increases cell proliferation and neurogenesis in the adult mouse dentate gyrus. Nat Neurosci 2:266-270

Wei Q, Hebda-Bauer EK, Pletsch A et al (2007) Overexpressing the glucocorticoid receptor in forebrain causes an aging-like neuroendocrine phenotype and mild cognitive dysfunction. J Neurosci 27:8836-8844
Westberry JM, Sadosky PW, Hubler TR, Gross KL, Scammell JG (2006) Glucocorticoid resistance in squirrel monkeys results from a combination of a transcriptionally incompetent glucocorticoid receptor and overexpression of the glucocorticoid receptor cochaperone FKBP51. J Steroid Biochem Mol Biol 100: 34-41

Wochnik GM, Rüegg J, Abel GA, Schmidt U, Holsboer F, Rein $\mathrm{T}$ (2005) FK506-binding proteins 51 and 52 differentially regulate dynein interaction and nuclear translocation of the glucocorticoid receptor in mammalian cells. J Biol Chem 280:4609-4616 